EISSN: 2706-7955 ISSN: 2077-4605

DOI: 10.36632/mejar/2021.10.3.58

Journal homepage: www.curresweb.com

Pages: 852-865

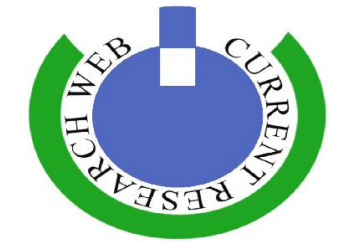

\title{
Pedological features of some Western Delta soils, Egypt and their relationships with different taxonomic systems
}

Zayed A.M.A., Al-Toukhy A.A. and El-Tapey H.M.A.

Soils, Water and Environment Research Institute, Agricultural Research Center, Giza, Egypt

Received: 10 July $2021 \quad$ Accepted: 30 July $2021 \quad$ Published: 10 August 2021

\begin{abstract}
Soil Western Delta under consideration is situated between latitudes $30^{\circ} 10^{\prime} 00^{\prime \prime}$ and $30^{\circ} 37^{\prime} 30^{\prime \prime}$ North and longitudes $30^{\circ} 05^{\prime} 00^{\prime \prime}$ and $30^{\circ} 47^{\prime} 30^{\prime \prime}$ "East and comprises an area of about 274,540.9 feddans. The pedological features and soil properties were studied according to delineation of physiographic units using remote sensing techniques. There are two main physiographic units as follows : 1) River terraces which includes two sub-units, i.e., the oldest river terraces, and river terraces of deltaic stage, these sub-units soil represent about 28.82 and $24.15 \%$ of the total area, respectively. 2) Wadi El-Natrun complex which includes five sub-units, i.e., transition soils, gullies soils, windblown soils (soils of sand dunes), isolated plateau, and water body (lake depression ), these soils occupy about 21.30, 14.11, $5.81,1.90$ and $3.90 \%$ of the total area. The current study were carried out by three classification systems, i.e., USDA, (2014), FAO ( 2006 ) and FAO ( 2015) and discussed some relationships between the previous classification systems which reflects the importance of applying both FAO and USDA systems to cover most of soil properties. We belief that both systems are in complementary.
\end{abstract}

Keywords: Physiographic units, Soil classification, USDA (2014), FAO (2006), FAO (2014 update 2015)

\section{Introduction}

A soil survey determines the characteristics of the soils in a given area, classifies the soil according to a standard system of classification, plots the boundaries of the soils on a map, and makes predictions about the behavior of soils. The different uses of the soils and how the response of management effects them are considered. The information collected in a soil survey helps in the development of land use plans and evaluates and predicts the effects of land use on the environment (Soil Survey Staff, 1993).

Wadi EL- Natrun depression occupies a natural excavation area and a defined physiographic characteristic in the Western desert. It is considered as a part of the old deltaic plain located west of the present Nile Delta. It is about $50 \mathrm{~km}$ in length and about $27 \mathrm{~km}$ in width. The lowest point in this Wadi is about $23 \mathrm{~m}$ below sea level (Harga and Elwan, 1978).

\subsection{Climate}

The climatological data of Wadi El Natrun station, (Table 1), reveal to relative humidity values are between $44 \%$ in May and $63 \%$ in November with annual mean is $54 \%$. Temperature mean varies between $13.3 \mathrm{C}^{\circ}$ in January and $27.6 \mathrm{C}^{\circ}$ in August. The highest temperature is $35.9 \mathrm{C}^{\circ}$ in August too and the lowest is $7.5 \mathrm{C}^{\circ}$ in January. The annual mean temperature is $21.0 \mathrm{C}^{\circ}$. The annual total precipitation is $41.4 \mathrm{~mm}$. The highest values is $13.0 \mathrm{~mm}$ which recorded in November, while June, July and August months haven't any rainfall. Evaporation values range from $5.2 \mathrm{~mm} /$ day in December and $14.0 \mathrm{~mm}$ in June, while the annual mean is $9.5 \mathrm{~mm} /$ day. The mean wind speed varies between 8.1 knots in both January and December and 11.4 knots in April, with annual mean 8.7 knots. 
Data of soil temperature, rainfall and evaporation aspiration reveal to the soil moisture regime is Torric or Aridic and the soil temperature regime is Thermic according to USDA (2014).

Table 1: Climatological data of Wadi El- Natrun Station (1946 - 1975).

\begin{tabular}{|c|c|c|c|c|c|c|c|}
\hline \multirow{2}{*}{ Month } & \multirow{2}{*}{$\begin{array}{c}\text { Relative } \\
\text { humidity \% }\end{array}$} & \multicolumn{3}{|c|}{ Temperature $\left(\mathrm{C}^{0}\right)$} & \multirow{2}{*}{$\begin{array}{c}\text { Rainfall } \\
(\mathrm{mm})\end{array}$} & \multirow{2}{*}{$\begin{array}{c}\text { Evaporation } \\
\text { per day }(\mathrm{mm})\end{array}$} & \multirow{2}{*}{$\begin{array}{c}\text { Mean wind } \\
\text { speed } \\
\text { (Knots) }\end{array}$} \\
\hline & & Max. & Min. & Mean & & & \\
\hline January & 61 & 20.1 & 7.5 & 13.3 & 6.7 & 5.3 & 8.1 \\
\hline February & 54 & 21.4 & 8.2 & 14.4 & 3.9 & 6.8 & 9.1 \\
\hline March & 50 & 24.6 & 9.8 & 17.1 & 3.5 & 8.7 & 10.5 \\
\hline April & 48 & 28.7 & 12.7 & 20.4 & 1.9 & 10.9 & 11.4 \\
\hline May & 44 & 32.4 & 16.0 & 23.5 & 1.1 & 12.7 & 10.6 \\
\hline June & 47 & 35.1 & 19.1 & 26.6 & 0.0 & 14.0 & 10.6 \\
\hline July & 53 & 35.6 & 20.4 & 27.3 & 0.0 & 13.2 & 10.0 \\
\hline August & 55 & 35.9 & 20.8 & 27.6 & 0.0 & 12.1 & 10.0 \\
\hline September & 58 & 33.6 & 18.9 & 25.5 & 0.3 & 10.5 & 9.2 \\
\hline October & 57 & 30.5 & 16.3 & 22.8 & 0.6 & 8.6 & 8.7 \\
\hline November & 63 & 25.8 & 12.8 & 18.7 & 13.0 & 6.3 & 8.3 \\
\hline December & 61 & 21.5 & $9 . .2$ & 14.7 & 10.4 & 5.2 & 8.1 \\
\hline Total & - & - & - & - & 41.4 & - & - \\
\hline Annual mean & 54 & 28.8 & 14.3 & 21.0 & - & 9.5 & 8.7 \\
\hline
\end{tabular}

\subsection{Geology}

According to Shata et al. (1962) and La Moreaux (1962) Quaternary lake deposits and old alluvial deposits are the main features which cover the studied area. The Quaternary deposits are underlain sedimentary rocks of Pleistocene and Pliocene ages which consist of sand and gravel interbedded with thin layers of clay. These, in turn, are underlain by limestone of Miocene, Oligocene and Paleocene ages. Beds of chalk and limestone of Cretaceous and Eocene ages are brought to the surface in the northward direction. Said (1990) stated that Wadi EL-Natrun formation includes the marine carbonate-shale sequence of middle Jurassic age. The carbonates of the section are mostly dolomitic and are more frequent in the upper part of the section. Anhydrite is present in the Gebel Rissu and Kattaniya wells. The Wadi El-Natrun is always overlain by the Khatatba formation. The Wadi ElNatrun formation includes more limestone beds in its top part than the lower part of the overlying Khatatba formation.

\subsection{Water resources}

According to Abdel Salam (1968), the main feeding source for the ground water aquifer in the studied area is restricted to the Nile and Delta region, whereas, agriculture would be totally dependent on the ground water.

The aim of the study under consideration is to throw some light on the physiographic features and the main soil units were selected by using remote sensing techniques. Application some systems of soil taxonomy to choice the more suitable mean for exposure the soil properties of soil evaluation characteristics for irrigation by using parametric method and selection more suitable crops. Some systems of soil taxonomy were applied to choice the more suitable mean for exposure the soil properties of soil evaluation characteristics for irrigation by using parametric method and selection more suitable crops.

\section{Materials and Methods}

The area under consideration is located in the western desert adjacent to the alluvial soils of Western Nile Delta. It is situated between latitudes $30^{\circ} 10^{\prime} 00^{\prime \prime}$ and $30^{\circ} 37^{\prime} 30^{\prime \prime}$ North and longitudes $30^{\circ} 05^{\prime} 00^{\prime \prime}$ and $30^{\circ} 47^{\prime} 30^{\prime \prime}$ East and comprises an area of about 274540.9 feddans. Pre-field work, researches were started by training on soil survey methodology, collection data and information about 
the study area i.e. geology, topography, land resources..etc, then detailed investigations were applied through digital image processing and classification to define the physiographic units and their classes.

\subsection{Physiographic analysis:}

By using digital image data of 2010 and its processing of Landsat Enhanced Thematic Mapper plus (ETM + ) image having a frame between path 176 and row 39 which were executed using ENVI 4.7 software ITT (2009). The different landform were initially determined from the satellite image and the digital elevation model (DEM) was extracted from the contour map, following the methodology by Dobos et al. (2002) and Kalogirous (2002). Through digital image processing, the physiographic units are delineated and illustrated in Map 1 with representative soil profiles.

\subsection{Field Work:}

Check the validity and accuracy of boundaries was done as a first step of field observations by using many mini pits, followed by digging thirteen soil profiles to represent different physiographic units of the studied area which were delineated or observed by using the physiographic analysis. The morphological feature and profile description were undertaken according to the methods used by guidelines of FAO (2006 a) and are illustrated in Table 2.

\subsection{Analytical methods:}

The primary treatments on soil samples of different representative profiles were: organization, air drying, crushing and sieving samples through $2.0 \mathrm{~mm}$ sieve, there after both granulometric and chemical analyses procedures were carried according to the following:

\subsection{Physical properties:}

These properties were fulfilled according to Burt (2004) which included both particle size distribution and contents of organic matter, gypsum and calcium carbonate. The obtainable data were illustrated in Table 3.

\subsection{Chemical properties:}

All chemical properties were carried out in the soil saturation extract, i.e., electrical conductivity (EC) and soluble cations and anions except soluble sulfate anion which was calculated by subtraction total anions from total cations. The saturation percentage was determined during preparation the paste, while, soil reaction $(\mathrm{pH})$ was measured in the soil paste. These procedures were emphasized according to Burt (2004). The exchangeable sodium percentage (ESP) values were calculated from sodium adsorption ration (SAR) which determined by concentrations of soluble $\mathrm{Na}, \mathrm{Ca}$ and $\mathrm{Mg}$ according to Richared (1954). The obtainable data were illustrated in Table 4.

\subsection{Soil Classification:}

Three systems of classification were used in the current study, respectively USDA (2014), FAO (2006 b) and FAO (2015). 


\section{Results and Discussion}

The studied area occupies about 274540.9 feddans and covers two main physiographic units which were recognized as follows:

\begin{tabular}{|c|c|c|}
\hline \multirow{2}{*}{ Physiographic mapping units } & \multicolumn{2}{|c|}{ Area in } \\
\hline & Feddan & $\%$ \\
\hline \multicolumn{3}{|c|}{ First : river terraces } \\
\hline 1.1 - The oldest river terraces & 79124.2 & 28.82 \\
\hline 1.2 - River terraces of deltaic stage & 66314.9 & 24.15 \\
\hline \multicolumn{3}{|c|}{ Second : Wadi El-Natrun complex } \\
\hline 2.1 - Transition soils & 58478.6 & 21.30 \\
\hline 2.2 - Gullies soils & 38736.1 & 14.11 \\
\hline 2.3 - Windblown soils (soils of sand dunes) & 15957.6 & 5.81 \\
\hline 2.4 - Isolate plateau & 5229.5 & 1.90 \\
\hline 2.5 - Water body ( Lake depression ) & 10700.0 & 3.90 \\
\hline Total Area & 274540.9 & 100.00 \\
\hline
\end{tabular}

Soils representing the main physiographic units are discussed here in:

\subsection{The river terraces:}

The soils of river terraces occupy a large portion of the eastern part of the studied area and they are distinctly a part of the old river delta formation. Three terraces can easily be recognized, which are characterized by a difference in elevation, the oldest terrace highest and the youngest lowest (UNDP/FAO, 1963). The studied area represents the oldest river terraces and river terraces of deltaic stage. The soils of these terraces are deep to very deep, common to abundant gravelly coarse sands, usually are more or less dissected by erosion gullies which give the area an undulating they are barren and in generally appear present desert pavement. Soil characteristics of different terraces as follows:

\subsubsection{The oldest river traces.}

These soils cover the largest portion of the studied area about $28.82 \%$ of total area and are represented by soil profiles 1, 2 and 3. Data in Table 2 clear that topography of the studied profiles differ between almost flat and gently undulating, on the other hand, slope is between nearly level and gently sloping surrounding the studied profiles. Native vegetation are absent around representative soil profiles 1 and 2, while there are scattering shrubs around the third representative one.

All representative profiles are deep $>100 \mathrm{~cm}$. Gravel contents vary between 10 and $55 \%$ by volume and tend to decrease with depth except soil of profile 3 has an opposite trend. Soil texture classes tend to roughness with depth and change from sandy loam to sand. Soil structure differs between loose and massive. The important pedogenic features are presence of few gypsum segregations in all representative profiles and few lime segregations in soil of profile 1 only. Data of physical properties in Table 3 reveal to coarse sand is the predominant constituents and differs between 62.25 and $87.92 \%$, follows by silt, clay and fine sand, respectively, in the first two representative profiles, while in the third profile contents of fine sand $>$ silt $>$ clay. Contents of fine sand are between 2.59 and $14.85 \%$. Silt and clay contents are from 3.77 to $15.12 \%$ and from 1.12 to $11.99 \%$, respectively. Total carbonate tends to increase with depth and its contents change between 4.23 and $9.30 \%$. Gypsum contents vary from 4.82 to $12.90 \%$ and tend to increase with depth except soils of profile 2, which have an opposite trend. Organic matter (O.M) contents tend to decrease with depth and represent trace constituents between 0.08 and $0.21 \%$.

The chemical properties that are important for describing and identifying soils are illustrated in Table 4. Soil reaction $(\mathrm{pH})$ varies between 7.29 and 7.80, i.e., it fluctuates between neutral and slightly alkaline as a descriptive terms. Soil salinity changes widely between 4.73 and $45.08 \mathrm{dS} / \mathrm{m}$, which corresponds to slightly and strongly saline classes, respectively. Strongly saline level is related to soils of profile 3. Generally, salinity tends to increase with depth in this unit and soluble sodium cation is a predominant constituent in profile 3 . Soluble sulfate is the relevant anion in the first two profiles, while in the third is soluble chloride. Values of sodium adsorption ratio (SAR) and 
exchangeable sodium percentage (ESP) clear no sodicity of profiles 1 and 2, while soils of profile 3 have sodic effect.

According to USDA (2014) soils of representative profiles may be classified as follows:

- Typic Calcigypsids, sandy, mixed, thermic.

- Leptic Haplogypsids, sandy, mixed, thermic.

- Leptic Haplogypsids, sandy-skeletal, mixed thermic.

On the other hand classify according to FAO (2006 b) as follows:

- Hypogypsic Gypsisols (Aridic, Arenic).

- Hypogypsic Gypsisols (Yermic, Arenic).

- Endosalic Hypogypsic Gypsisols (Sodic, Yermic, Skeletic, Arenic). and classify according to FAO (2015) as follows:

- Haplic Gypsisols (Arenic, Hypogypsic, Aridic).

- Haplic Gypsisols (Arenic, Hypogypsic, Yermic).

(profile 1)

(profile 2)

- Skeletic Gypsisols (Arenic, Hypogypsic, Endosalic, Sodic, Yermic). (profile 3)

\section{1.2. River terraces of deltaic stage.}

These soils consist of material brought in by the Nile but deposited under the shallow water of the bay or estuary into which the generally narrow Nile Valley opened out (UNDP/FAO, 1963).

These soils occupy about $24.15 \%$ or fifth of the studied area and are represented by soil profiles 4 and 5. Data in Table 2 show that the soils around the representative profiles have almost flat topography and nearly level slope. The native vegetation observed only on soils without desert pavements as shrubs. Many gravel are observed on the surface forming a kind of desert pavement. Gravel contents through different layers differ widely between 5 and $40 \%$ (by volume) may be due to variations in river discharge and sea level. The pedogenic features fluctuate between absence and few lime and gypsum segregations in all layers of representative profiles or some layers.

Physical properties in Table 3 show that, sand is the texture class and the coarse sand is the predominant constituent, except layer $40-60 \mathrm{~cm}$ of profile 4 , and changes from 42.02 to $94.16 \%$. Fine sand contents follow coarse sand and differ between 2.16 and $51.70 \%$. Fine fractions of silt and clay consider trace constituents and are from 1.88 to $8.89 \%$ and 1.14 to $3.17 \%$, respectively. Total carbonate contents tend to be homogenous in each profile separately and record values between 2.11 and $5.92 \%$. Gypsum contents vary from 0.86 to $6.88 \%$, deep layer of profile 4 has higher content. Organic matter contents change between 0.07 and $0.27 \%$. It is considered as a trace constituent and tends to decrease with depth. Data in Table 4 show that, soil reaction ranges between Neutral (7.10) and moderately alkaline (8.10) and tends to increase with depth, which is affected by salinity contents in soils of profile 4 and has an opposite trend in soils of profile 5 . Soil salinity differs widely from $2.30 \mathrm{dS} / \mathrm{m}$ (very slightly saline) to $30.36 \mathrm{dS} / \mathrm{m}$ (strongly saline) and tends to decrease with depth. Distribution of soluble ions follows: $\mathrm{Na}^{+}>\mathrm{Ca}^{2+}>\mathrm{Mg}^{2+}>\mathrm{K}^{+}$and $\mathrm{Cl}^{-}>\mathrm{SO}_{4}{ }^{2-}>\mathrm{HCO}_{3}$, generally, in soils of profile 4. Soils of profile 5 haven't soluble cations trend, while soluble anions appear $\mathrm{SO}_{4}{ }^{2-}>\mathrm{Cl}>\mathrm{HCO}_{3}{ }^{-}$. Different layers of representative profiles don't show soil sodicity except upper two layers of profile 4 .

Application of different taxonomic systems on representative profiles shows: USDA (2014):

- Sodic Haplogypsids, sandy, mixed, thermic.

- Typic Torripsamments, siliceous, thermic.

(profile 4)

FAO (2006 b):

- Hypogypsic Gypsisols (Sodic, Yermic ,Arenic).

- Haplic Arenosols (Calcaric, Yermic ). FAO (2015) :

(profile 4)

(profile 5)

- Haplic Gypsisols (Arenic, Hypogypsic, Sodic, Yermic).

(profile 4) 
- Calcaric Arenosols (Yermic).

(profile 5)

\subsection{Wadi El-Natrun complex}

\subsubsection{Transition soils.}

Transition soils lie between river terraces and Wadi El-Natrun. Their origin may be traced to the erosion of the original Pliocene deposits and the renewed deposition of sands in the early Pleistocene, and the gravel or sand in the middle Pleistocene (UNDP/FAO, 1963).

These soils occupy about $21.30 \%$ of the study area and are represented by soil profiles $6,7,8$ and 9 (Map 1). Most of them are cultivated with fruit crops such as mango and olive and vegetables such as tomato.

Morphological description in Table 2 reveals to almost flat topography and nearly level slope. Absence or decrease of gravel contents on the surface may be due to manmade, while soil of profile 9 appears barren of native vegetation may be due to presence of a desert pavement of different gravel size. Practical size distribution shows coarse texture classes between sand and sandy loam. Gravel content through different layers of representative profiles changes from 5 and $40 \%$. The main pedogenic features is presence of few or very few lime segregations, except soils of profile 8 and subsurface layers of profile 9 . The same quantities of gypsum segregations are observed in the surface layer of profiles 6 and 9. Data of physical properties (Table 3) show that coarse sand is the predominant constituent and ranges between 55.69 and $88.12 \%$ followed by fine sand, silt and clay, respectively, except in soil profile 9 where silt $>$ clay $>$ fine sand \%. Total carbonate differs between 1.69 and 7.61 $\%$ and tends to decrease with depth. Gypsum contents vary from 0.86 to 6.19 and tend to decrease with depth too and the same trend is observed in organic matter contents, which change from 0.04 to 0.34 $\%$. Chemical properties in Table (4) show that soil reaction $(\mathrm{pH})$ is between 7.15 (neutral) and 8.37 (moderately alkaline). Soil salinity as EC values range from non-saline $(1.5 \mathrm{dS} / \mathrm{m})$ to moderately saline $(15.20 \mathrm{dS} / \mathrm{m})$, which decrease with depth, and surface layers have higher contents. Generally, distribution of soluble cations follows sequence: $\mathrm{Na}^{+}>\mathrm{Ca}^{2+}>\mathrm{Mg}^{2+}>\mathrm{K}^{+}$and distribution of soluble anions follows: $\mathrm{Cl}>\mathrm{SO}_{4}{ }^{2-}>\mathrm{HCO}_{3}{ }^{-}$. Surface layer of profiles $6,7 \& 8$, and deepest layer of profile 7 recorded values of SAR and ESP higher than 13 and 15, respectively, which are related to sodicity effect.

Application of USDA (2014), FAO (2006 b) and FAO (2015) systems, respectively, soils are classified as follows:

USDA (2014)

- Typic Torriorthents, sandy-skeletal, mixed, thermic.

- Typic Torripsamments, siliceous, thermic.

- Typic Torriorthents, sandy, mixed, thermic. FAO (2006 b)

- Haplic Solonchaks (Sodic, Chloridic, Aridic, Arenic).

- Protic Arenosols (Calcaric, Hyposalic, Aridic).

- Haplic Regosols (Calcaric, Hyposalic, Yermic, Arenic). FAO (2015)

- Sodic Solonchaks (Arenic, Chloridic, Calcaric, Skeletic, Aridic).

- Sodic Solonchaks (Arenic, Chloridic, Calcaric, Aridic).

- Calcaric Protic Sodic Arenosols (Aridic).

- Calcaric Protic Regosols (Arenic, Yermic). (profiles 6, 7)

(profile 8)

(profile 9)

(profile 6)

(profile 8)

(profile 9)

(Profile 6)

(Profile 7)

(profile 8)

(profile 9)

\subsubsection{Gullies soils.}

These soils are gently undulating to steeply dissect by erosion and they have many wide, but not always deep gullies filled with wind-blown sand (Harga and Elwan, 1978). These soils occupy about $14.11 \%$ of total area. Field observations (Table, 2) show that these soils have almost flat topography, nearly level slope, haven't native vegetation and have desert pavement of medium and coarse pebbles and stones . Gravel through representative profile 10 or any pedogenic features are not observed. Data of physical properties show that these soils have loamy sand texture class in surface layer and sand 


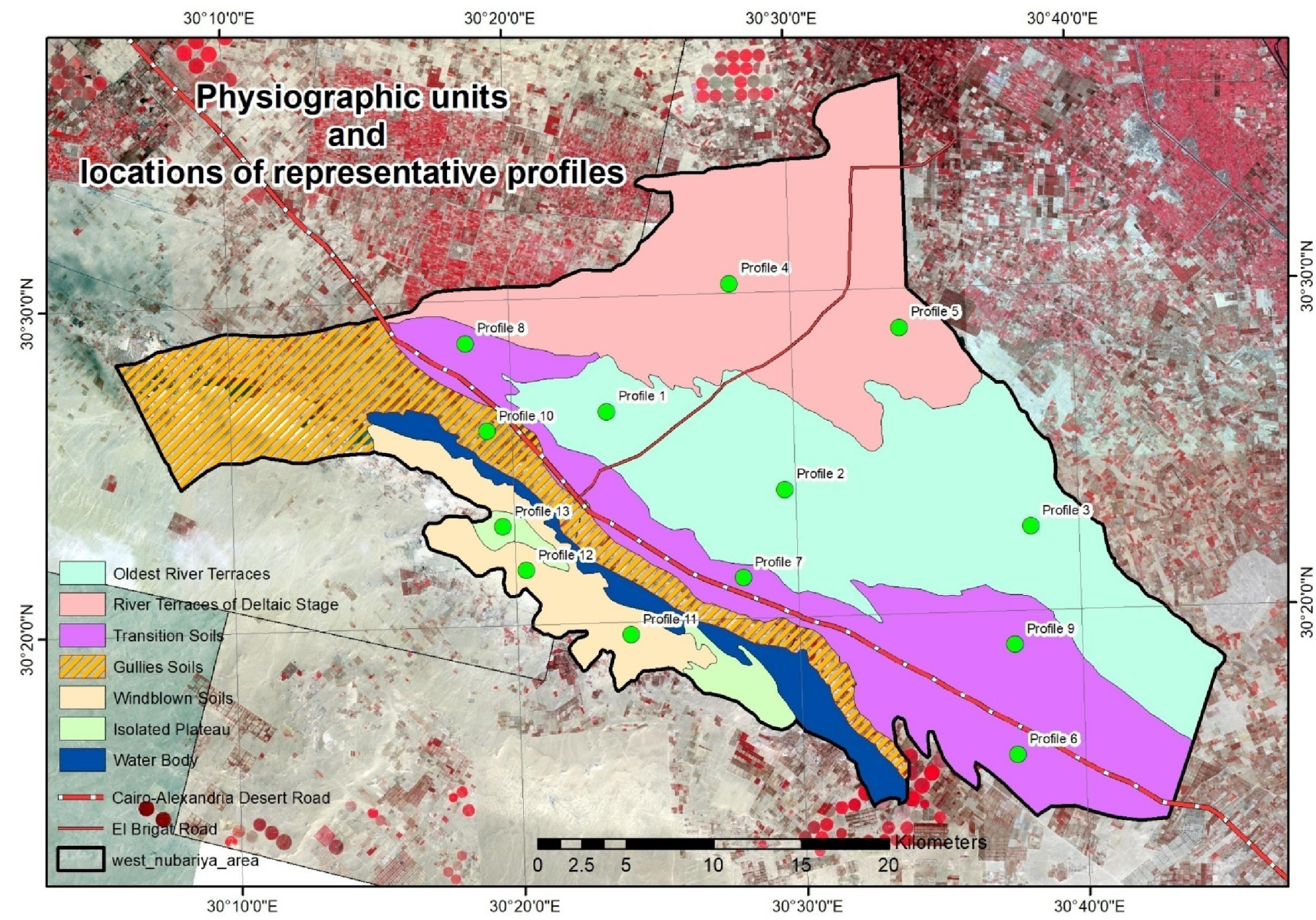

Map 1: Physiographic units and locations of the representative profiles. 
Table 2: Morphological description of the representative soil profiles.

\begin{tabular}{|c|c|c|c|c|c|c|c|c|c|c|c|c|c|c|c|c|c|c|}
\hline \multirow{2}{*}{ 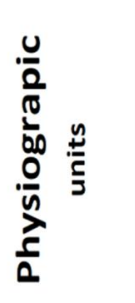 } & \multirow[b]{2}{*}{$\begin{array}{l}\dot{0} \\
2 \\
0 \\
\frac{1}{2} \\
\dot{0} \\
0\end{array}$} & \multirow[b]{2}{*}{ 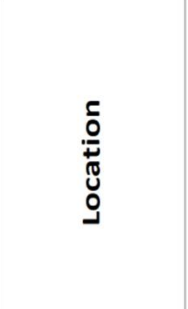 } & \multirow[b]{2}{*}{$\begin{array}{l}\frac{1}{2} \\
\frac{0}{\pi} \\
\frac{0}{00} \\
0 \\
0 \\
0\end{array}$} & \multirow[b]{2}{*}{$\frac{0}{\frac{0}{n}}$} & \multirow[b]{2}{*}{ 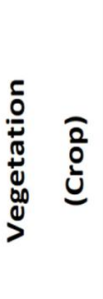 } & \multirow[b]{2}{*}{ 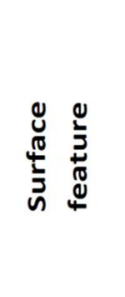 } & \multirow[b]{2}{*}{ 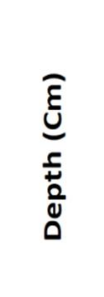 } & \multicolumn{2}{|c|}{ Colour } & \multirow[b]{2}{*}{$\begin{array}{l}\overline{0} \\
\sum_{\pi}^{0} \\
\overline{0}\end{array}$} & \multirow[b]{2}{*}{ 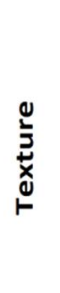 } & \multirow[b]{2}{*}{ 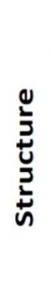 } & \multicolumn{2}{|c|}{ Consistence } & \multirow{2}{*}{$\begin{array}{l}\text { Pedogenic } \\
\text { Feature }\end{array}$} & \multirow[b]{2}{*}{$\begin{array}{l}n \\
0 \\
0 \\
x\end{array}$} & \multirow[b]{2}{*}{ 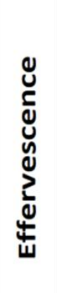 } & \multirow[b]{2}{*}{$\begin{array}{l}\frac{1}{2} \\
\frac{\pi}{0} \\
\frac{1}{3} \\
0 \\
0 \\
0\end{array}$} \\
\hline & & & & & & & & Dry & Moist & & & & $\vec{t}$ & $\frac{\ddot{n}}{0}$ & & & & \\
\hline \multicolumn{19}{|c|}{ River Terraces } \\
\hline \multirow[t]{3}{*}{$\begin{array}{l}\text { Oldest } \\
\text { river } \\
\text { terraces }\end{array}$} & 1 & $\begin{array}{l}30^{\circ} 24^{\circ} 14^{=}=\mathrm{N} \\
30^{\circ} 22^{\circ} 45^{=} \mathrm{E}\end{array}$ & $\mathrm{AF}$ & NL & Barren & $\begin{array}{l}\text { Few } \\
\text { coarse } \\
\text { gravel }\end{array}$ & $\begin{array}{l}0-25 \\
25-60 \\
60-100\end{array}$ & $\begin{array}{l}10 \text { YR } 7 / 6 \\
7.5 \text { YR } 6 / 8 \\
7.5 \text { YR } 5 / 6 \\
\end{array}$ & $\begin{array}{l}10 \text { YR } 6 / 6 \\
7.5 \text { YR } 5 / 8 \\
7.5 \text { YR } 4 / 6\end{array}$ & $\begin{array}{l}40 \\
35 \\
10\end{array}$ & $\begin{array}{l}\text { SL } \\
\text { SL } \\
\text { LS }\end{array}$ & $\begin{array}{l}M \\
M \\
M\end{array}$ & $\begin{array}{l}S \\
S \\
S\end{array}$ & $\begin{array}{l}\mathrm{VF} \\
\mathrm{VF} \\
\mathrm{VF}\end{array}$ & $\begin{array}{l}\text { Few lime \& gypsum segregations } \\
\text { Few lime \& gypsum segregations } \\
\text { Few lime \& gypsum segregations }\end{array}$ & $\begin{array}{c}\text { V.F.F. } \\
\text { V.F.F. } \\
-.\end{array}$ & $\begin{array}{l}+++ \\
+++ \\
+++\end{array}$ & $\begin{array}{l}\text { Gw } \\
\text { Gw }\end{array}$ \\
\hline & 2 & $\begin{array}{l}30^{\circ} 23^{\circ} 46^{=}=\mathrm{N} \\
30^{\circ} 23^{\circ} 38^{=}=E\end{array}$ & AF & NL & Barren & $\begin{array}{l}\text { Anundant } \\
\text { different } \\
\text { gravel } \\
\text { size }\end{array}$ & $\begin{array}{l}0-50 \\
50-110\end{array}$ & $\begin{array}{l}7.5 \text { YR } 6 / 8 \\
7.5 \text { YR } 7 / 8\end{array}$ & $\begin{array}{l}7.5 \text { YR } 5 / 8 \\
7.5 \text { YR } 6 / 8\end{array}$ & 40 & LS & SG & L & L & $\begin{array}{l}\text { Few gypsum segregations } \\
\text { Few gypsum segregations }\end{array}$ & $\begin{array}{l}\text { V.F.F. } \\
\text { V.F.F. }\end{array}$ & +++ & Gw \\
\hline & 3 & $\begin{array}{l}30^{\circ} 25^{\circ} 00^{=} \mathrm{N} \\
30^{\circ} 24^{-} 57^{=}=\mathrm{E}\end{array}$ & GU & GS & $\begin{array}{l}\text { Schutt- } \\
\text { ering } \\
\text { shrubs }\end{array}$ & $\begin{array}{l}\text { Anundant } \\
\text { different } \\
\text { gravel } \\
\text { size }\end{array}$ & $\begin{array}{l}0-50 \\
50-100\end{array}$ & $\begin{array}{l}10 \text { YR } 7 / 6 \\
10 \text { YR } 7 / 6\end{array}$ & $\begin{array}{l}10 \text { YR } 6 / 6 \\
10 \text { YR } 6 / 6\end{array}$ & 30 & $S$ & $M$ & Sh & VF & $\begin{array}{l}\text { Few gypsum segregations } \\
\text { Few gypsum segregations }\end{array}$ & $\begin{array}{c}\text { V.F.F. } \\
--\end{array}$ & +++ & CW \\
\hline \multirow[t]{2}{*}{$\begin{array}{l}\text { River } \\
\text { tarraces of } \\
\text { deltaic } \\
\text { stage }\end{array}$} & 4 & $\begin{array}{l}30^{\circ} 25^{\circ} 20^{=}=\mathrm{N} \\
30^{\circ} 26^{-} 22^{=}=\mathrm{E}\end{array}$ & AF & NL & $\begin{array}{l}\text { Many } \\
\text { shrubs }\end{array}$ & $\begin{array}{l}\text { Many } \\
\text { medium } \\
\text { gravel }\end{array}$ & $\begin{array}{l}0-20 \\
20-40 \\
40-60 \\
60-100\end{array}$ & $\begin{array}{l}10 \text { YR } 6 / 8 \\
10 \text { YR } 6 / 6 \\
10 \text { YR } 6 / 8 \\
10 \text { YR } 7 / 4 \\
\end{array}$ & $\begin{array}{l}10 \text { YR } 5 / 8 \\
10 \text { YR } 5 / 6 \\
10 \text { YR } 5 / 8 \\
10 \text { YR } 6 / 4\end{array}$ & $\begin{array}{c}40 \\
35 \\
5 \\
5\end{array}$ & $\begin{array}{l}S \\
S \\
S \\
S\end{array}$ & $\begin{array}{l}M \\
M \\
M \\
M\end{array}$ & $\begin{array}{l}S \\
S \\
S \\
S\end{array}$ & $\begin{array}{l}\mathrm{VF} \\
\mathrm{VF} \\
\mathrm{VF} \\
\mathrm{VF}\end{array}$ & $\begin{array}{l}\text { Few lime \& gypsum segregations } \\
\text { Few lime segregations } \\
\text { Few lime \& gypsum segregations }\end{array}$ & \begin{tabular}{c|} 
F.F \\
M.F \\
M.F \\
.- \\
\end{tabular} & $\begin{array}{l}+++ \\
+++ \\
+++ \\
+++\end{array}$ & $\begin{array}{l}\text { Gw } \\
\text { Cw } \\
\text { Gw } \\
--\end{array}$ \\
\hline & 5 & $\begin{array}{l}30^{\circ} 26^{\circ} 13^{=}=\mathrm{N} \\
30^{\circ} 28^{-} 26^{\prime}=\mathrm{E}\end{array}$ & $\mathrm{AF}$ & NL & $\begin{array}{c}\text { Few } \\
\text { shrubs }\end{array}$ & $\begin{array}{l}\text { many } \\
\text { different } \\
\text { gravel } \\
\text { size }\end{array}$ & $\begin{array}{l}0-20 \\
20-55 \\
55-120\end{array}$ & $\begin{array}{l}10 \text { YR } 7 / 4 \\
10 \text { YR } 7 / 3 \\
10 \text { YR } 7 / 4\end{array}$ & $\begin{array}{l}10 \text { YR } 6 / 4 \\
10 \text { YR } 6 / 3 \\
10 \text { YR } 6 / 4\end{array}$ & $\begin{array}{l}35 \\
40 \\
10\end{array}$ & $\begin{array}{l}S \\
S \\
S\end{array}$ & $\begin{array}{l}\text { SG } \\
M \\
M\end{array}$ & $\begin{array}{l}\text { L } \\
\text { Sh } \\
\text { Sh }\end{array}$ & $\begin{array}{l}\text { L } \\
\text { V.F } \\
\text { V.F }\end{array}$ & 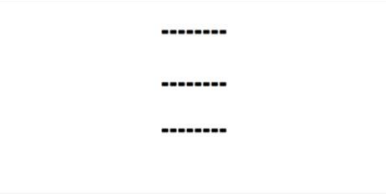 & $\begin{array}{l}\text { F.F } \\
-- \\
\text { F.F }\end{array}$ & $\begin{array}{l}+++ \\
+++ \\
+++\end{array}$ & $\begin{array}{l}\text { Gw } \\
\text { CW }\end{array}$ \\
\hline
\end{tabular}


Table 2 : Cont.

\begin{tabular}{|c|c|c|c|c|c|c|c|c|c|c|c|c|c|c|c|c|c|c|}
\hline \multirow{2}{*}{ 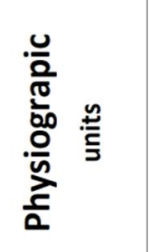 } & \multirow[b]{2}{*}{ 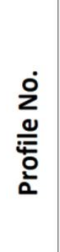 } & \multirow[b]{2}{*}{ 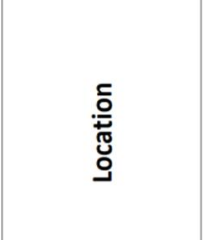 } & \multirow[b]{2}{*}{ 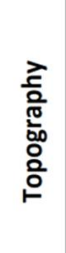 } & \multirow[b]{2}{*}{$\frac{\check{0}}{\text { 은 }}$} & \multirow[b]{2}{*}{ 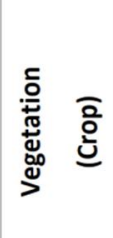 } & \multirow[b]{2}{*}{ 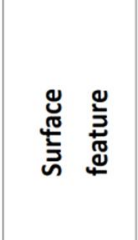 } & \multirow[b]{2}{*}{ 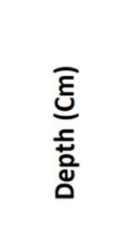 } & \multicolumn{2}{|c|}{ Colour } & \multirow[b]{2}{*}{ गे } & \multirow[b]{2}{*}{ 站 } & \multirow[b]{2}{*}{ 总 } & \multicolumn{2}{|c|}{ Consistence } & \multirow{2}{*}{$\begin{array}{l}\text { Pedogenic } \\
\text { Feature }\end{array}$} & \multirow[b]{2}{*}{$\stackrel{2}{\circ}$} & \multirow[b]{2}{*}{ 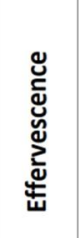 } & \multirow[b]{2}{*}{ 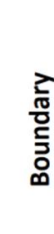 } \\
\hline & & & & & & & & Dry & Moist & & & & ฉ̀ & 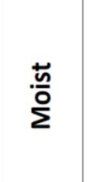 & & & & \\
\hline \multicolumn{19}{|c|}{ Wadi EL-Natrun } \\
\hline \multirow[t]{4}{*}{$\begin{array}{c}\text { Transition } \\
\text { soils }\end{array}$} & 6 & $\begin{array}{cc}30^{\circ} & 17^{-} 38^{=} \mathrm{N} \\
30^{\circ} & 27^{-} 35^{\prime}=\mathrm{E}\end{array}$ & AF & NL & Mango & $\begin{array}{c}\text { Few } \\
\text { medium } \\
\text { gravel }\end{array}$ & $\begin{array}{c}0-45 \\
45-100 \\
\end{array}$ & \begin{tabular}{|l|}
10 YR $6 / 6$ \\
10 YR $7 / 8$ \\
\end{tabular} & $\begin{array}{l}10 \text { YR } 5 / 6 \\
10 \text { YR } 6 / 8 \\
\end{array}$ & 40 & $\mathrm{~s}$ & $\begin{array}{l}\text { SG } \\
\text { SG }\end{array}$ & $\mathrm{L}$ & $\mathrm{L}$ & $\begin{array}{l}\text { Very few lime \& gypsum } \\
\text { segregations } \\
\text { Very few lime concretions }\end{array}$ & $\begin{array}{l}\text { F.F. } \\
\text { M.F } \\
\end{array}$ & $\begin{array}{l}+++ \\
+++ \\
++\end{array}$ & $\begin{array}{c}\text { Gw } \\
--\end{array}$ \\
\hline & 7 & $\begin{array}{l}30^{\circ} 21^{-} 38^{=}=\mathrm{N} \\
30^{\circ} 23^{-} 37^{=} \mathrm{E}\end{array}$ & AF & NL & Olive & $\begin{array}{c}\text { Few } \\
\text { medium } \\
\text { gravel }\end{array}$ & $\begin{array}{c}0-20 \\
20-70 \\
70-120\end{array}$ & \begin{tabular}{|l|}
10 YR $5 / 4$ \\
10 YR $6 / 8$ \\
10 YR $6 / 6$ \\
\end{tabular} & $\begin{array}{l}10 \text { YR } 4 / 4 \\
10 \text { YR } 5 / 8 \\
10 \text { YR } 5 / 6 \\
\end{array}$ & \begin{tabular}{|c|}
10 \\
35 \\
5 \\
\end{tabular} & $\begin{array}{l}\mathrm{S} \\
\mathrm{S} \\
\mathrm{S}\end{array}$ & $\begin{array}{l}M \\
M \\
M\end{array}$ & $\begin{array}{c}\mathrm{S} \\
\mathrm{S} \\
\mathrm{Sh}\end{array}$ & $\begin{array}{l}\text { V.F } \\
\text { V.F } \\
\text { V.F }\end{array}$ & $\begin{array}{l}\text { Few lime segregations } \\
\text { Few lime segregations } \\
\text { Few lime segregations }\end{array}$ & $\begin{array}{c}\text { F.F. } \\
\text { M.F } \\
-- \\
\end{array}$ & $\begin{array}{l}++ \\
+++ \\
+++\end{array}$ & $\begin{array}{c}\mathrm{CW} \\
\mathrm{CW} \\
--\end{array}$ \\
\hline & 8 & $\begin{aligned} 30^{\circ} 27^{-} 09 & =\mathrm{N} \\
30^{\circ} 19^{\circ} 43^{-} & =\mathrm{E}\end{aligned}$ & AF & NL & Tomato & -- & $\begin{array}{c}0-20 \\
20-100\end{array}$ & \begin{tabular}{|l|}
10 YR $7 / 4$ \\
10 YR $7 / 4$ \\
\end{tabular} & $\begin{array}{l}10 \text { YR } 6 / 4 \\
10 \text { YR } 6 / 4\end{array}$ & $\begin{array}{l}5 \\
5\end{array}$ & $\begin{array}{l}\mathrm{S} \\
\mathrm{S}\end{array}$ & $\begin{array}{l}M \\
M\end{array}$ & $\begin{array}{l}\mathrm{s} \\
\mathrm{s}\end{array}$ & $\begin{array}{l}\text { V.F } \\
\text { V.F }\end{array}$ & ------ & $\begin{array}{l}\text { F.F. } \\
\text { V.F.F. }\end{array}$ & $\begin{array}{l}+++ \\
+++\end{array}$ & $\begin{array}{c}\mathrm{Gw} \\
--\end{array}$ \\
\hline & 9 & $\begin{array}{l}30^{\circ} 16^{-} 20^{=}=\mathrm{N} \\
30^{\circ} 30^{-} 20^{\prime}=\mathrm{E}\end{array}$ & AF & NL & Barren & $\begin{array}{c}\text { Anundant } \\
\text { different } \\
\text { gravel } \\
\text { size }\end{array}$ & $\begin{array}{c}0-12 \\
12-35 \\
35-95 \\
95-150 \\
\end{array}$ & \begin{tabular}{|l|}
7.5 YR $6 / 4$ \\
10 YR $8 / 4$ \\
10 YR $8 / 2$ \\
7.5 YR $7 / 4$ \\
\end{tabular} & $\begin{array}{l}7.5 \text { YR } 5 / 4 \\
10 Y R 7 / 4 \\
10 Y R 7 / 2 \\
7.5 Y R 6 / 4 \\
\end{array}$ & \begin{tabular}{l|}
40 \\
10 \\
10 \\
10 \\
\end{tabular} & $\begin{array}{l}\text { SL } \\
\text { SL } \\
\text { S } \\
\text { S }\end{array}$ & $\begin{array}{l}\text { M } \\
M \\
\text { SG } \\
\text { SG }\end{array}$ & $\begin{array}{l}\text { Sh } \\
\text { Sh } \\
\mathrm{L} \\
\mathrm{L}\end{array}$ & \begin{tabular}{l|}
$F$ \\
$F$ \\
$L$ \\
$L$
\end{tabular} & $\begin{array}{c}\text { Few lime \& gypsum segregations } \\
------ \\
------ \\
---- \\
\end{array}$ & $\begin{array}{l}-- \\
-- \\
-- \\
--\end{array}$ & $\begin{array}{l}+++ \\
+++ \\
+++ \\
+++\end{array}$ & $\begin{array}{l}\mathrm{CW} \\
\mathrm{CW} \\
\mathrm{GW}\end{array}$ \\
\hline $\begin{array}{c}\text { Gullies } \\
\text { soils }\end{array}$ & 10 & $\begin{array}{l}30^{\circ} 25^{\circ} 00^{\circ}=\mathrm{N} \\
30^{\circ} 25^{\circ} 00^{\circ}=\mathrm{E}\end{array}$ & AF & NL & Barren & $\begin{array}{l}\text { Many } \\
\text { stones }\end{array}$ & $\begin{array}{c}0-30 \\
30-100\end{array}$ & \begin{tabular}{|l|}
10 YR $6 / 4$ \\
10 YR $7 / 4$ \\
\end{tabular} & $\begin{array}{l}10 \text { YR } 5 / 4 \\
10 \text { YR } 6 / 4 \\
\end{array}$ & $\begin{array}{l}-- \\
--\end{array}$ & $\begin{array}{c}\text { LS } \\
\text { S }\end{array}$ & $\begin{array}{l}M \\
S G\end{array}$ & $\begin{array}{l}\mathrm{S} \\
\mathrm{L}\end{array}$ & $\begin{array}{c}\text { V.F } \\
L\end{array}$ & ------ & $\begin{array}{l}-- \\
--\end{array}$ & $\begin{array}{l}+++ \\
+++\end{array}$ & $\begin{array}{c}\mathrm{CW} \\
--\end{array}$ \\
\hline \multirow[t]{2}{*}{$\begin{array}{c}\text { Windblown } \\
\text { Soils }\end{array}$} & 11 & $\begin{array}{l}30^{\circ} 22^{\circ} 03^{=}=\mathrm{N} \\
30^{\circ} 21^{-} 26^{=}=\mathrm{E}\end{array}$ & AF & NL & $\begin{array}{c}\text { Few } \\
\text { shrubs }\end{array}$ & $-\cdots$ & \begin{tabular}{|c|}
$0-40$ \\
$40-120$ \\
\end{tabular} & \begin{tabular}{|l|}
10 YR $8 / 4$ \\
10 YR $8 / 8$ \\
\end{tabular} & $\begin{array}{l}10 \mathrm{YR} 7 / 4 \\
10 \mathrm{YR} 7 / 8 \\
\end{array}$ & $\begin{array}{l}-- \\
--\end{array}$ & $\begin{array}{l}\mathrm{S} \\
\mathrm{S}\end{array}$ & $\begin{array}{l}\text { SG } \\
\text { SG } \\
\end{array}$ & $\begin{array}{l}\mathrm{L} \\
\mathrm{L}\end{array}$ & $\begin{array}{l}\mathrm{L} \\
\mathrm{L}\end{array}$ & ------ & $\begin{array}{c}\text { V.F } \\
--\end{array}$ & $\begin{array}{l}+++ \\
+++\end{array}$ & Gw \\
\hline & 12 & 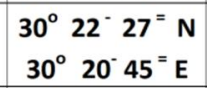 & AF & NL & $\begin{array}{c}\text { Few } \\
\text { shrubs }\end{array}$ & $\begin{array}{l}\text { Humman } \\
\text { artificats }\end{array}$ & \begin{tabular}{|c|}
$0-50$ \\
$50-120$ \\
\end{tabular} & \begin{tabular}{|l|}
10 YR $7 / 3$ \\
10 YR $8 / 4$ \\
\end{tabular} & $\begin{array}{l}10 \text { YR } 6 / 3 \\
10 \text { YR } 7 / 4\end{array}$ & $\begin{array}{l}- \\
--\end{array}$ & $\begin{array}{l}\mathrm{S} \\
\mathrm{S}\end{array}$ & $\begin{array}{l}\text { SG } \\
\text { SG }\end{array}$ & $\begin{array}{l}\mathrm{L} \\
\mathrm{L}\end{array}$ & $\begin{array}{l}\mathrm{L} \\
\mathrm{L}\end{array}$ & $\begin{array}{l}\text { Lamela } \\
\text { Lamela }\end{array}$ & \begin{tabular}{|c|} 
V.F.F. \\
--
\end{tabular} & $\begin{array}{l}+++ \\
+++\end{array}$ & $\begin{array}{c}\mathrm{Gw} \\
--\end{array}$ \\
\hline $\begin{array}{l}\text { Isolated } \\
\text { plateau }\end{array}$ & 13 & $\begin{array}{l}30^{\circ} 23^{-} 01^{=}=\mathrm{N} \\
30^{\circ} 21^{-} 01^{\prime}=\mathrm{E}\end{array}$ & $A F$ & NL & $\begin{array}{l}\text { Very } \\
\text { few } \\
\text { shrubs }\end{array}$ & $\begin{array}{c}\text { Few } \\
\text { medium } \\
\text { gravel }\end{array}$ & $\begin{array}{c}0-40 \\
40-55 \\
55-100\end{array}$ & \begin{tabular}{|l|}
$2.5 \times 7 / 2$ \\
$2.5 \times 6 / 2$ \\
$2.5 \times 6 / 4$
\end{tabular} & $\begin{array}{l}2.5 \text { Y } 6 / 2 \\
2.5 \text { Y } 5 / 2 \\
2.5 \text { Y } 5 / 4\end{array}$ & $\begin{array}{l}-- \\
-- \\
--\end{array}$ & \begin{tabular}{|c|}
$S$ \\
$S L$ \\
$S C L$
\end{tabular} & $\begin{array}{l}\text { SG } \\
M \\
M\end{array}$ & $\begin{array}{c}\mathrm{L} \\
\mathrm{Sh} \\
\mathrm{H}\end{array}$ & $\begin{array}{l}\mathrm{L} \\
\mathrm{F} \\
\mathrm{Fi}\end{array}$ & $\begin{array}{l}\text { Few hard lime nodules } \\
\text { Many hard lime nodules } \\
\text { Many hard lime nodules }\end{array}$ & $\begin{array}{l}- \\
-- \\
--\end{array}$ & 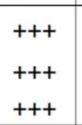 & $\begin{array}{c}\text { AW } \\
\text { GW } \\
--\end{array}$ \\
\hline \multicolumn{19}{|c|}{ Topography: AF = Almost flat } \\
\hline $\begin{array}{l}\text { Texture: SiL } \\
\text { Structure: N } \\
\text { Roots: F.F }\end{array}$ & $\begin{array}{l}=\text { Sil } \\
\mathrm{M}=\mathrm{N} \\
=\mathrm{FeW}\end{array}$ & 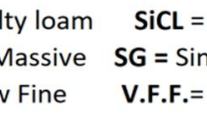 & $\begin{array}{l}\text { Silty } C^{\prime} \\
\text { gle Gr } \\
\text { Very }\end{array}$ & $\begin{array}{l}\text { Clay L } \\
\text { irains } \\
\text { Few F }\end{array}$ & $\begin{array}{l}\text { Loam SL } \\
\text { Consist } \\
\text { Fine }\end{array}$ & $\begin{array}{l}\text { = Sandy Lo } \\
\text { stence, (Dr } \\
\text { Mf = Many }\end{array}$ & $\begin{array}{l}\text { y) }: \text { CL }=\text { Ch }=\text { Slig } \\
\text { Fine }\end{array}$ & $\begin{array}{l}\text { Clay Loam } \\
\text { shtly hard }\end{array}$ & $\begin{array}{l}\mathbf{S}=\text { Sand } \mathbf{L S} \\
\mathbf{H}=\text { Hard } \mathbf{L} \\
\text { Boundary: }\end{array}$ & & & $\begin{array}{l}\text { Sand } \\
\mathbf{S}= \\
\text { dual }\end{array}$ & $\begin{array}{l}=\text { Soft } \\
\text { Wavy }\end{array}$ & $\begin{array}{l}=\text { Sand } \\
\text { (Moist } \\
\text { CW }=\end{array}$ & $\begin{array}{l}\text { d Clay Loam V.F.S }=\text { Very Fine Sand } \\
\text { st) : } \quad \mathbf{F}=\text { Friable } \quad \mathbf{F}=\text { Firm } \mathbf{V F} \\
=\text { Clear Wavy } \mathbf{D}=\text { Diffuse }\end{array}$ & $\begin{array}{l}=V_{e} \\
=A b r\end{array}$ & $\begin{array}{l}\text { raible } \\
\text { Wavy }\end{array}$ & \\
\hline
\end{tabular}


Table 3 : Physical properties of the representative soil profiles.

\begin{tabular}{|c|c|c|c|c|c|c|c|c|c|c|c|}
\hline \multirow{2}{*}{$\begin{array}{c}\text { Physiographic } \\
\text { units }\end{array}$} & \multirow{2}{*}{$\begin{array}{l}\text { Profile } \\
\text { No. }\end{array}$} & \multirow{2}{*}{$\begin{array}{l}\text { Depth } \\
\text { cm. }\end{array}$} & \multicolumn{5}{|c|}{ Particle size distribution ( $\%$ ) } & \multirow{2}{*}{$\begin{array}{c}\text { Texture } \\
\text { class }\end{array}$} & \multirow{2}{*}{$\begin{array}{c}\mathrm{CaCO}_{3} \\
\%\end{array}$} & \multirow{2}{*}{$\begin{array}{c}\text { Gypsum } \\
\%\end{array}$} & \multirow{2}{*}{$\begin{array}{c}\text { O.M } \\
\%\end{array}$} \\
\hline & & & $\begin{array}{c}\text { Coarse } \\
\text { sand }\end{array}$ & $\begin{array}{l}\text { Fine } \\
\text { Sand }\end{array}$ & $\begin{array}{l}\text { Total } \\
\text { sand }\end{array}$ & Silt & Clay & & & & \\
\hline \multicolumn{12}{|l|}{ River Terraces } \\
\hline \multirow[t]{7}{*}{ Oldest river terraces } & \multirow[t]{3}{*}{1} & $0-25$ & 62.25 & 10.64 & 72.89 & 15.12 & 11.99 & SL & 6.76 & 4.82 & 0.21 \\
\hline & & $25-60$ & 70.65 & 7.00 & 77.65 & 13.08 & 9.27 & SL & 8.82 & 4.82 & 0.11 \\
\hline & & $60-100$ & 75.62 & 5.37 & 80.99 & 10.87 & 8.14 & $\overline{L S}$ & 9.30 & $\mathbf{7 . 7 4}$ & 0.09 \\
\hline & \multirow[t]{2}{*}{2} & $0-50$ & 77.37 & 3.63 & 81.00 & 9.13 & 9.87 & LS & 6.34 & 9.80 & 0.19 \\
\hline & & $50-110$ & 85.55 & 2.59 & 88.14 & 9.97 & 1.89 & $\mathbf{S}$ & 4.65 & 6.88 & 0.08 \\
\hline & \multirow[t]{2}{*}{3} & $0-50$ & 87.92 & 7.19 & 95.11 & 3.77 & 1.12 & $\mathbf{S}$ & 4.23 & 11.18 & 0.17 \\
\hline & & $50-100$ & 79.27 & 14.85 & 94.12 & 3.87 & 2.01 & $\mathbf{S}$ & 5.07 & 12.90 & 0.11 \\
\hline \multirow{7}{*}{$\begin{array}{l}\text { River terraces of } \\
\text { deltic stage }\end{array}$} & \multirow[t]{4}{*}{4} & $0-20$ & 78.27 & 9.67 & $\mathbf{8 7 . 9 4}$ & 8.89 & 3.17 & $\mathbf{S}$ & 5.07 & 5.16 & 0.27 \\
\hline & & $20-40$ & 88.35 & 6.17 & 94.52 & 3.27 & 2.21 & $\mathbf{S}$ & 3.38 & 2.41 & 0.13 \\
\hline & & $40-60$ & 42.02 & 51.70 & 93.72 & 3.32 & 2.96 & $\mathbf{S}$ & 5.07 & 2.41 & 0.07 \\
\hline & & $60-100$ & 90.87 & 5.24 & 96.11 & 1.88 & 2.01 & $\mathbf{s}$ & 5.92 & 6.88 & 0.07 \\
\hline & \multirow[t]{3}{*}{5} & $0-20$ & 93.82 & 2.16 & 95.98 & 2.01 & 2.01 & $\mathbf{S}$ & 2.53 & 2.58 & 0.26 \\
\hline & & $20-55$ & 90.62 & 4.42 & 95.04 & 3.19 & 1.77 & $\mathbf{S}$ & 2.11 & 1.72 & 0.18 \\
\hline & & $55-120$ & 94.16 & 2.18 & 96.34 & 2.52 & 1.14 & $\mathbf{s}$ & 2.53 & 0.86 & 0.07 \\
\hline \multicolumn{12}{|l|}{ Wadi EL-Natrun } \\
\hline \multirow[t]{11}{*}{ Transition soils } & \multirow[t]{2}{*}{6} & $0-45$ & 80.05 & 12.95 & 93.00 & 3.88 & 3.12 & $\mathbf{S}$ & 5.92 & 4.82 & 0.34 \\
\hline & & $45-100$ & 83.32 & 11.50 & 94.82 & 3.02 & 2.16 & $\mathbf{S}$ & 3.38 & 1.38 & 0.21 \\
\hline & \multirow[t]{3}{*}{7} & $0-20$ & 72.12 & 21.22 & 93.34 & 2.92 & 3.74 & $\mathbf{S}$ & 7.61 & 3.44 & 0.33 \\
\hline & & $20-70$ & 71.38 & 23.48 & 94.86 & 1.95 & 3.19 & $\mathbf{S}$ & 7.61 & 4.13 & 0.18 \\
\hline & & $70-120$ & 72.18 & 13.84 & 86.02 & 8.11 & 5.87 & $\mathbf{S}$ & 5.92 & 2.41 & 0.19 \\
\hline & \multirow[t]{2}{*}{8} & $0-20$ & 85.30 & 9.74 & 95.04 & 2.77 & 2.19 & $\mathbf{s}$ & 3.80 & 0.86 & 0.23 \\
\hline & & $20-100$ & 88.12 & 6.53 & 94.65 & 4.26 & 1.09 & $\mathbf{s}$ & 3.38 & 0.86 & 0.14 \\
\hline & \multirow[t]{4}{*}{9} & $0-12$ & 62.30 & 10.11 & 72.41 & 14.95 & 12.64 & $\mathbf{S L}$ & 6.76 & 6.19 & 0.11 \\
\hline & & $12-35$ & 55.69 & 13.99 & 69.68 & 16.18 & 14.14 & SL & 1.69 & 1.72 & 0.09 \\
\hline & & $35-95$ & 78.05 & 3.44 & 81.49 & 13.40 & 5.11 & LS & 2.11 & 1.55 & 0.07 \\
\hline & & $95-150$ & 60.65 & 6.85 & 67.50 & 18.20 & 14.30 & SL & 2.53 & 0.86 & 0.04 \\
\hline \multirow[t]{2}{*}{ Gullies soils } & \multirow[t]{2}{*}{10} & $0-30$ & 69.22 & 14.80 & 84.02 & 11.76 & 4.22 & LS & 4.23 & 1.72 & 0.36 \\
\hline & & $30-100$ & 84.92 & 7.32 & 92.24 & 4.64 & 3.12 & $\mathbf{s}$ & 3.38 & 1.72 & 0.12 \\
\hline Windblown Soils & 11 & $0-40$ & 92.82 & 2.62 & 95.44 & 3.12 & 1.44 & $\mathbf{s}$ & 3.38 & 3.27 & 0.25 \\
\hline & & $40-120$ & 92.97 & 1.17 & 94.14 & 2.77 & 3.09 & $\mathbf{S}$ & 2.53 & 1.72 & 0.14 \\
\hline & 12 & $0-50$ & 91.25 & 5.45 & 96.70 & 1.59 & 1.71 & $\mathbf{s}$ & 3.38 & 1.72 & 0.28 \\
\hline & & $50-120$ & 90.80 & 5.97 & 96.77 & 1.22 & 2.01 & $\mathbf{s}$ & 2.96 & 4.30 & 0.15 \\
\hline Isolated plateau & 13 & $0-40$ & 90.60 & 7.12 & 97.72 & 1.14 & 1.14 & $\mathbf{S}$ & 8.46 & 1.38 & 0.37 \\
\hline & & $40-55$ & 66.02 & 8.53 & 74.55 & 15.24 & 10.21 & $\mathbf{S L}$ & 48.64 & 0.52 & 0.24 \\
\hline & & $55-100$ & 37.67 & 20.11 & 57.78 & 21.78 & 20.44 & Sal & 32.15 & 0.69 & 0.19 \\
\hline
\end{tabular}


Middle East J. Agric. Res., 10(3): 852-865, 2021

Table 4 : Chemical properties of the representative soil profiles.

\begin{tabular}{|c|c|c|c|c|c|c|c|c|c|c|c|c|c|c|c|}
\hline \multirow{2}{*}{$\begin{array}{c}\text { Physiographic } \\
\text { units }\end{array}$} & \multirow{2}{*}{$\begin{array}{c}\text { Profile } \\
\text { No. }\end{array}$} & \multirow{2}{*}{$\begin{array}{l}\text { Depth } \\
\text { cm. }\end{array}$} & \multirow[b]{2}{*}{ SP } & \multirow[b]{2}{*}{ pH } & \multirow{2}{*}{$\begin{array}{c}\mathbf{E C} \\
\mathrm{dS} / \mathrm{m} .\end{array}$} & \multicolumn{4}{|c|}{ Cations me./l } & \multicolumn{4}{|c|}{ Anions me./I } & \multirow[b]{2}{*}{ SAR } & \multirow[b]{2}{*}{ ESP } \\
\hline & & & & & & $\mathbf{C a}^{++}$ & $\mathbf{M g}^{++}$ & $\mathbf{N a}^{+}$ & $\mathbf{K}^{+}$ & $\mathrm{CO}_{3}=$ & $\mathrm{HCO}_{3}$ & $\mathbf{C l}^{-}$ & $\mathbf{S O}_{4}=$ & & \\
\hline \multicolumn{16}{|c|}{ River Terraces } \\
\hline \multirow{7}{*}{ Oldest river terraces } & \multirow[t]{3}{*}{$\overline{1}$} & $0-25$ & 34 & 7.29 & 4.95 & 26.32 & 3.03 & 20.35 & 3.04 & 0.0 & 1.65 & 19.49 & 31.59 & 5.31 & 6.17 \\
\hline & & $25-60$ & 33 & 7.32 & 4.73 & 27.63 & 4.98 & 20.35 & 0.12 & 0.0 & 0.94 & 13.56 & 38.57 & 5.04 & 5.81 \\
\hline & & $60-100$ & 24 & 7.36 & 5.00 & 21.05 & 16.99 & 22.96 & 0.25 & 0.0 & 1.18 & 26.27 & 33.80 & 5.26 & 6.11 \\
\hline & \multirow[t]{2}{*}{2} & $0-50$ & 21 & 7.38 & 7.02 & 38.16 & 23.80 & 33.04 & 2.79 & 0.0 & 1.65 & 38.14 & 58.00 & 5.94 & 6.97 \\
\hline & & $50-110$ & 18 & 7.51 & 8.62 & 44.74 & 9.61 & 33.91 & 1.26 & 0.0 & 1.65 & 31.36 & 56.52 & 6.51 & 7.69 \\
\hline & \multirow[t]{2}{*}{3} & $0-50$ & 19 & 7.80 & 20.24 & 98.68 & 64.36 & 140.00 & 0.71 & 0.0 & 1.42 & 254.24 & 48.10 & 15.51 & 17.77 \\
\hline & & $50-100$ & 23 & 7.70 & 45.08 & 185.53 & 86.21 & 340.00 & 1.37 & 0.0 & 1.42 & 508.47 & 103.22 & 29.17 & 29.46 \\
\hline \multirow{7}{*}{$\begin{array}{l}\text { River terraces of } \\
\text { deltaic stage }\end{array}$} & \multirow[t]{4}{*}{4} & $0-20$ & 22 & 7.79 & $\mathbf{3 0 . 3 6}$ & 134.21 & 39.70 & 160.00 & 1.97 & 0.0 & 1.89 & \begin{tabular}{|l|}
296.61 \\
\end{tabular} & 37.39 & 17.16 & 19.39 \\
\hline & & $20-40$ & 21 & 7.82 & 13.95 & 55.26 & 37.13 & 102.61 & 1.06 & 0.0 & 1.89 & 186.44 & 7.73 & 15.10 & 17.36 \\
\hline & & $40-60$ & 21 & 7.86 & 8.51 & 43.42 & 7.67 & 37.20 & 0.61 & 0.0 & 1.18 & 44.92 & 42.80 & 7.36 & 8.76 \\
\hline & & $60-100$ & 23 & 8.10 & 2.30 & 3.95 & 2.57 & 17.59 & 0.91 & 0.0 & 1.18 & 10.17 & 13.67 & 9.74 & 11.59 \\
\hline & \multirow[t]{3}{*}{5} & $0-20$ & 24 & 7.52 & 3.82 & 11.18 & 4.03 & 12.52 & 8.92 & 0.0 & 1.18 & $\mathbf{8 . 4 7}$ & 27.00 & 4.54 & 5.16 \\
\hline & & $20-55$ & 22 & 7.20 & 2.01 & 7.89 & 7.32 & 5.57 & 0.21 & 0.0 & 1.42 & 5.93 & 13.65 & 2.02 & 1.69 \\
\hline & & $55-120$ & 23 & 7.10 & 3.61 & 13.16 & 16.19 & 8.30 & 0.24 & 0.0 & 1.18 & 9.32 & 27.39 & 2.17 & 1.90 \\
\hline \multicolumn{16}{|c|}{ Wadi EL-Natrun } \\
\hline \multirow[t]{11}{*}{ Transition soils } & \multirow[t]{2}{*}{$\overline{76}$} & $0-45$ & 23 & $\mathbf{7 . 4 5}$ & 15.20 & 87.28 & 21.41 & 116.67 & 0.47 & 0.0 & 0.32 & 183.61 & 41.90 & 15.83 & 18.09 \\
\hline & & $45-100$ & 22 & 7.25 & 2.30 & 10.53 & 2.52 & \begin{tabular}{|c|}
10.83 \\
\end{tabular} & 1.32 & 0.0 & 0.94 & 17.80 & 6.46 & 4.24 & 4.76 \\
\hline & \multirow[t]{3}{*}{7} & $0-20$ & 16 & 7.15 & 15.07 & 53.16 & 28.15 & 104.00 & 2.07 & 0.0 & 0.85 & \begin{tabular}{|l|}
157.93 \\
\end{tabular} & 28.60 & 16.31 & 18.57 \\
\hline & & $20-70$ & 15 & 7.87 & 11.06 & 39.47 & 20.31 & 66.96 & 3.55 & 0.0 & 1.89 & 91.69 & 36.71 & 12.25 & 14.39 \\
\hline & & $70-120$ & 19 & 7.97 & 14.15 & 42.11 & 23.11 & 85.00 & 2.84 & 0.0 & 1.42 & 107.12 & 44.52 & 14.89 & 17.15 \\
\hline & 8 & $0-20$ & 18 & 8.37 & 4.52 & 11.18 & 1.86 & 36.00 & 1.32 & 0.0 & 1.89 & 29.66 & 18.81 & 14.10 & 16.34 \\
\hline & & $20-100$ & 19 & 8.00 & 1.99 & 2.63 & 3.35 & 15.48 & 0.63 & 0.0 & 1.42 & 19.49 & 1.18 & 8.95 & 10.67 \\
\hline & 9 & $0-12$ & 30 & 7.90 & 10.87 & $\mathbf{5 3 . 9 5}$ & 12.36 & 56.52 & 2.13 & 0.0 & 1.89 & 72.88 & 50.18 & 9.82 & 11.68 \\
\hline & & $12-35$ & 31 & 7.89 & 3.80 & 13.16 & 14.02 & $\begin{array}{l}11.68 \\
\end{array}$ & 0.50 & 0.0 & 1.42 & 10.17 & 27.77 & 3.17 & 3.30 \\
\hline & & $35-95$ & 26 & 7.64 & 1.50 & 6.58 & 7.55 & 3.02 & 0.50 & 0.0 & 1.42 & 7.63 & 8.61 & 1.13 & 0.41 \\
\hline & & $95-150$ & 34 & 7.68 & 2.65 & 7.89 & 7.32 & 13.37 & $\mathbf{0 . 3 5}$ & 0.0 & 1.18 & 16.10 & 11.66 & 4.85 & 5.56 \\
\hline Gullies soils & 10 & $0-30$ & 27 & 7.26 & 23.46 & 39.47 & 14.87 & 225.00 & 4.10 & 0.0 & 1.65 & 195.76 & 86.04 & 43.16 & 38.43 \\
\hline & & $30-100$ & 22 & 7.59 & 13.71 & 39.47 & 9.44 & \begin{tabular}{|c|}
93.00 \\
\end{tabular} & 0.86 & 0.0 & 1.65 & 73.73 & 67.39 & 18.81 & 20.93 \\
\hline Windblown Soils & 11 & $0-40$ & 24 & 7.70 & 8.05 & 52.63 & 12.59 & 25.57 & 1.32 & 0.0 & 1.18 & 21.19 & 69.73 & 4.48 & 5.07 \\
\hline & & $40-120$ & 23 & 7.81 & 4.59 & 13.16 & 20.54 & 14.21 & 2.63 & 0.0 & 1.65 & 26.27 & 22.62 & 3.46 & 3.70 \\
\hline & 12 & $0-50$ & 20 & $\mathbf{7 . 5 8}$ & 5.57 & 32.89 & 4.06 & $\mathbf{1 7 . 5 9}$ & 2.03 & 0.0 & 1.89 & 22.03 & 32.65 & 4.09 & 4.56 \\
\hline & & $50-120$ & 20 & 7.68 & 3.86 & 26.32 & 4.12 & 9.14 & 1.42 & 0.0 & 1.65 & 7.63 & 31.72 & 2.34 & 2.15 \\
\hline Isolated plateau & 13 & $0-40$ & 26 & 7.62 & 14.39 & $\mathbf{1 4 . 4 7}$ & 7.27 & 126.00 & 3.65 & 0.0 & 1.65 & 53.39 & 96.35 & 38.22 & 35.53 \\
\hline & & $40-55$ & 36 & 7.74 & 32.43 & 3.95 & 6.92 & 336.00 & 3.70 & 0.0 & 1.42 & 169.49 & 179.66 & 144.13 & 67.88 \\
\hline & & $55-100$ & 46 & 7.63 & 136.28 & 5.26 & 6.69 & 1240.0 & 13.43 & 0.0 & 1.89 & $\mathbf{1 1 3 9 . 8 3}$ & 123.67 & 507.15 & 88.19 \\
\hline
\end{tabular}


within subsurface one. Particle size distribution follows sequence: coarse sand $>$ fine sand $>$ silt $>$ clay content. Total carbonate, gypsum and organic matter contents represent a trace constituent. Total carbonate records $4.23 \%$ in surface layer and $3.38 \%$ in the subsurface one. Gypsum content is $1.72 \%$ through the representative profile. Organic matter content is $0.36 \%$ in the surface layer and $0.12 \%$ in subsurface one. Chemical properties of the representative profile in Table 4 reveal to neutral reaction in surface layer, while slightly alkaline in the subsoil. Soil salinity records strongly saline class (23.46 $\mathrm{dS} / \mathrm{m})$ in surface layer and moderately saline one $(13.71 \mathrm{dS} / \mathrm{m})$ in subsurface. Distribution of soluble cations follows $\mathrm{Na}^{+}>\mathrm{Ca}^{2+}>\mathrm{Mg}^{2+}>\mathrm{K}^{+}$and soluble anions follows: $\mathrm{Cl}^{-}>\mathrm{SO}_{4}{ }^{2-}>\mathrm{HCO}_{3}-$. Values of both SAR and ESP reveal to the soil is affected by sodicity. These soils are mainly characterized by highly saline effect and classified according to USDA (2014) as:

- Typic Torriorthents, sandy, mixed, thermic.

While, it classified according to FAO (2006 b) and (2015) respectively as follows:

- Haplic Solonchaks (Sodic, Chloridic, Aridic, Arenic).

- Sodic Solonchaks (Arenic, Chloridic, Calcaric, Aridic).

\subsubsection{Windblown soils (soils of sand dunes).}

Soils of the depression would be formed on materials transported from hill tops and sides of old gullies. These soils represent about $5.81 \%$ of the total area. Soils of profiles 11 and 12 are the representative profiles which have almost flat topography, nearly level slope, few shrubs of native vegetation and no desert pavement. There are some human artifacts on the surface around profile 12 (Table 2). Physical properties, in Table (3) reveal to these soils have sand texture class and their particle size distribution follow two clusters: coarse sand $>$ silt $>$ fine sand $>$ clay in soil of profile 11 and coarse sand $>$ fine sand $>$ clay $>$ silt in soils of profile 12. Distribution of carbonate, gypsum and organic matter is similar in both representative profiles and their contents fluctuate from 2.53 to $3.38,1.72$ to $4.30 \%$ and 0.14 to $0.28 \%$, respectively. Soil chemical properties in Table 4 show that soil reaction expressed as $\mathrm{pH}$, ranges from slightly alkaline to moderately one. Values of $\mathrm{pH}$ tend to increase with depth. Soil salinity varies between 3.86 and $8.05 \mathrm{dS} / \mathrm{m}$, which corresponds very slightly saline and moderately saline class. Soil salinity tends to decrease with depth. Distribution of soluble cations follows the sequence: $\mathrm{Ca}^{2+}>\mathrm{Na}^{+}>\mathrm{Mg}^{2+}>\mathrm{K}^{+}$, except subsurface layer of profile 11 appears sequence: $\mathrm{Mg}^{2+}>$ $\mathrm{Na}^{+}>\mathrm{Ca}^{2+}>\mathrm{K},{ }^{+}$while, soluble anions follows : $\mathrm{SO}_{4}{ }^{2-}>\mathrm{Cl}^{-}>\mathrm{HCO}_{3}{ }^{-}$except subsurface layer of profile 11 which appear : $\mathrm{Cl}^{>}>\mathrm{SO}_{4}{ }^{2-}>\mathrm{HCO}_{3}{ }^{-}$. Both values of SAR and ESP don't reflect sodicity effect.

The soils under consideration may be classified for both representative profiles as follows: According to USDA (2014):

- $\quad$ Typic Torripsamments, siliceous, thermic.

According to FAO (2006 b):

- Protic Arenosols (Calcaric, Hyposalic, Aridic) \& Haplic Arenosols ( Calcaric, Hyposalic, Aridic ) .

According to FAO (2015):

- $\quad$ Calcaric Gypsiric Protic Arenosols (Aridic).

\subsubsection{Isolated plateau.}

These soils occur in plateaus of varying size and at different levels in the bottom of Wadi ElNatrun, mainly near the lake where they look like cliffs. These formations may be formed when the depression was still in open connection with the Pliocene Nile estuary. These soils occupy about 1.90 $\%$ of the study area and are represented by soil profile 13 . They have almost flat topography and nearly level slop on top with a predominantly rocky surface with few medium gravel and very few shrubs. Texture tends to be finer with depth. Sand texture is observed in surface layer, sandy loam in subsurface and sandy clay loam in deepest one. The main pedogenic feature is few to many hard lime nodules (Table 2). Particle size distribution reveals to coarse sand $>$ silt $>$ clay $>$ fine sand, except in surface layer whereas fine sand content $>$ silt clay, and their content as follows, respectively $37.67-90.60 \%$, $1.14-21.78 \% 1.14-20.44 \%$ and $7.12-20.11 \%$. These soils are rich in lime, which its content ranges between 8.46 and $48.64 \%$. Subsurface layer has higher content. Gypsum content isn't observed in the field and varies between 0.52 and $1.38 \%$. Organic matter content is a trace constituent and differs 
between 0.19 and $0.37 \%$, which decreases with depth (Table 3). Soil reaction is slightly alkaline whereas, $\mathrm{pH}$ values range between 7.62 and $7.74 \%$. Soil Salinity increases with depth and varies widely from moderately saline $(14.39 \mathrm{dS} / \mathrm{m})$ to strongly saline $(136.28 \mathrm{dS} / \mathrm{m})$. Location of the representative profile is adjacent to old lake and is affected by saline and alkaline conditions. Sodium is predominant constituent of soluble cations, while other cations are traces. Distribution of soluble anions follows: $\mathrm{SO}_{4}{ }^{+2}>\mathrm{Cl}^{-}>\mathrm{HCO}_{3}{ }^{-}$in upper two layered, while the deepest layer appears: $\mathrm{Cl}^{-}>\mathrm{SO}_{4}{ }^{2-}>\mathrm{HCO}_{3}{ }^{-}$. Both SAR and ESP values in all layers can reveal to sodicity effect. Soils of representative profile can be classified, respectively, according to USDA (2014), FAO (2006 b) and FAO (2015) as follows:

- Calcic Haplosalids, fine loamy, mixed, thermic.

- Calcic Hypersalic Solonchaks (Sodic, Chloridic, Aridic, Arenic).

- Calcic Sodic Solonchaks (Arenic, Loamic, Chloridic, Calcaric, Hypersalic, Aridic).

\subsubsection{Water body (Lake depression).}

The lowest point in the depression is $23 \mathrm{~m}$ below sea level and about $50 \mathrm{~m}$ below of its surrounding rim (Harga and Elwan, 1978).It fluctuates from season to another and represents about 3.90 $\%$ of the total area.

According to Zayed et al. (2020) which concluded and stated that it's preferable to apply USDA and FAO taxonomic systems in studies and reports may be due to compilation between them.

The current study applied the recent FAO system (2015) with applying both, systems USDA (2014) and FAO (2006 b). The FAO (2015) mentioned that the World Reference Base (WRB) is not intended to be a substitute for national soil classification systems, but rather to serve as denominator for communication at the international level. So, due to application of two FAO, systems in the study under consideration repeats the same conclusion of application both systems and add some observations:

FAO (2015) system use different sequences and different rules for using the qualifiers differs than 2006 system and supposed that are now suitable for both classifying soils and creating map legend. Several new qualifiers have been added to give more information about some important soil properties. The differences summary of the previous systems in the current study were illustrated in Table 5.

- Used term of "Protic" in Regosos reference Soil Groups showing no soil horizon development

- Gave an important presence of desert pavement in both FAO edition.

Table 5: Summary of the main observations around the applied systems of soil Classification in the current study.

\begin{tabular}{llll}
\hline Terms & FAO (2006)* & FAO (2015) & USDA (2014) \\
\hline $\begin{array}{l}\text { Levels of } \\
\text { category }\end{array}$ & $\begin{array}{l}\text { Two levels : } \\
\text {-Reference Soil Groups } \\
\text {-Qualifiers: Prefix \& } \\
\text { Suffix }\end{array}$ & $\begin{array}{l}\text { Two levels : } \\
\text {-Reference Soil Groups } \\
\text {-Qualifiers: Principal \& } \\
\text { Supplementary }\end{array}$ & $\begin{array}{l}\text { Five Categories : } \\
\text { Order, Suborders, Great } \\
\text { groups, Subgroups \& } \\
\text { Family }\end{array}$ \\
\hline Protic in Regosols & Not observed & $\begin{array}{l}\text { Is recorded as principal } \\
\text { qualifiers }\end{array}$ & $\begin{array}{l}\text { Entisols order have the } \\
\text { same meaning }\end{array}$ \\
\hline Yermic & Is recorded & Is recorded & Not observed \\
\hline $\begin{array}{l}\text { Carbonatic, Chloridic \& } \\
\text { Sulfatic as predominant } \\
\text { soluble anions }\end{array}$ & $\begin{array}{l}\text { Is recorded } \\
\text { In Solonchaks }\end{array}$ & $\begin{array}{l}\text { Is recorded } \\
\text { In Solonchaks }\end{array}$ & Not observed \\
\hline Skeletic in Solonchaks & Not observed & Is recorded & $\begin{array}{l}\text { Is recorded in family } \\
\text { level }\end{array}$ \\
\hline Calcaric in Solonchaks & Not observed & Is recorded & Not observed \\
\hline $\begin{array}{l}\text { Hyposalic in Arensols \& } \\
\text { Regosols }\end{array}$ & Is recorded & Not observed & . \\
\hline
\end{tabular}

*FAO (2006) is recorded in references as FAO (2006 b).

- Cared to identifying predominant soluble anion in Reference Soil Group (RSG) of "Solonchaks", i.e., Carbonatic, Chloridic and Sulfatic in both FAO edition.

- Used term "Skeletic" in RSG "Solonchaks", which show presence of coarse fragments in FAO 2015 edition. 
- $\quad$ Used term "Calcaric" in RSG "Solonchaks" in FAO 2015 edition.

- FAO (2006 b) appear salinity effect through term "Hyposalic" which reflects week level of this property, while it is not observed in system of FAO (2015), as noted in soil profiles 11 and12, i.e., FAO (2015) strike off term "Hyposalic", while, it retained two terms, "Hypocalcic" and "Hypogypsic".

Notice: FAO (2006 b) is the second edition of "World Reference Base", while, FAO (2014) is the third edition which update in 2015.

The current study observed some relationships between the applied classification systems of USDA, 2014, FAO, $2006 \mathrm{~b}$ and FAO, 2015. Also, gives an importance to application of both FAO and USDA systems to cover most of soil properties and give more detail information. We belief that both systems are in complementary.

\section{References}

Abdel Salam, M. A., 1968. Solis of Wadi El- Natrun area. Bulletin de I, Institut de D'esertd' Egypt, T. 16 Cairo.

ARE, 1979. "Climatological Normals for the Arab Republic of Egypt up to 1975. Printed in ARE by the General Organization for Gvot. Printing offices, Cairo.

Burt, R. E., 2004. "Soil Survey Laboratory Methods Manual". Soil Survey Investigation Report No. 42 Version 4.0 November.

Dobos, E., B. Norman, W. Bruee, M. Luca, J. Chris and M. Enka, 2002. The use of DEM and Satellite Images for Regional Scale Soil Database. $1^{\text {th }}$ World Congress of Soil Science (WCSS). 14 -21 August 2002, Bangkok, Thaili.

FAO, 2006 a." Guidelines for Soil Description" , 4 th Ed., FAO, Rome, Italy. ISBN: 92 -5-105521-1.

FAO, 2006 b. "World Reference Base for Soil Resources "A Framework for International classification, correlation and communication. World Soil Resources Reports.103, Rome, Italy.

FAO, 2015. "Word Reference Base for Soil Resources 2014" Update 2015. International soil classification system for naming soils and creating legends for soil map. World Soil Resources Reports 106, Rome, Italy.

Harga, A. A. and A.A. Elwan, 1978. Geomorphology and soil classification of Wadi El - Natrun area based on photo-interpretation. Desert Inst. Bull. ARE, 28, No.1, PP. 115 -131.

ITT, 2009. ITT corporation ENVI4.7 software 1133 Westchester Avenue, While plains, NY, 10604, USA.

Kalogirous, S., 2002. Expert Systems and GIS, An application of Land Suitability. Comput. Environ. Urban Syst. 26: 89 - 112.

La Moreaux, P.E., 1962. Reconnaissance report recommendations for ground water investigations, Wadi El- Natrun, Western Desert of Egypt. The General Desert Development Organization, Cairo, UAR.

Richared, L.A., 1954. "Diagnosis Improvement of Saline and Alkali Soils". U.S. Dept. Agric. Handbook No. 60, Washington, DC., USA.

Said, R., 1990. "The Geology of Egypt". Published by A.A. Balkema, Old Post Road, Brookfield, VT 05036, USDA.

Shata, A., M. Pavlov and K.F. Saad, 1962. The geology, hydrology of ground water, of Wadi ElNatrun. The General Desert Development Organization, Cairo, UAR.

Soil Survey Staff, 1993. "Soil Survey Manual". USDA Handbook 18 U.S. Government Printing office, Washington, D.C., USA.

UNDP/FAO, 1963. High Dam Soil Survey, United Arab Republic, Volume II, FAO /SF:16/UAR, The Reconnaissance Soil Survey, United Nations Development Program (Special Fund).

USDA, 2014. "Keys to Soil Taxonomy". 12 ${ }^{\text {th }}$ Edition, United States Department of Agriculture, Natural Resources Conservation Service, New York, USA.

Zayed, A. M. R., H. M. A. El-Tapey and A. A. Al-Toukhy, 2020. Study of some soils South El- Amiria, Alexandria Governorate, Egypt, using two soil classification systems. Egypt. J. Agric. Res., 98 (3): $548-558$. 